\title{
10 Jahre nach dem NSU. Vom Reden über Frauenhass und rechten Terror
}

\author{
Juliane Lang
}

Eingegangen: 19. Juli 2021 / Angenommen: 23. September 2021 / Online publiziert: 6. Dezember 2021

(C) Der/die Autor(en) 2021

Zusammenfassung Frauenfeindlichkeit und Frauenhass spielten eine relevante Rolle in rechtsterroristischen Anschlägen der vergangenen zehn Jahre. In der Folge fanden geschlechterrelevante Perspektiven zunehmend Eingang in das Reden über rechten Terror. Der Beitrag untersucht die widersprüchlichen Effekte des Redens über Frauenhass und rechten Terror in neueren Debatten. Nachgezeichnet wird eine Kontinuität des Verbesonderns rechter und frauenfeindlicher Gewalt sowie eine Entlastung der Mehrheitsgesellschaft von einer Mitverantwortung für rechten Terror.

Schlüsselwörter Rechter Terror · NSU · Antifeminismus · Frauenfeindlichkeit/ Frauenhass · Incels

\section{Talking about hatred for women and right-wing terror ten years after NSU}

\begin{abstract}
Misogyny and hatred for women played a relevant role in right-wing terrorist attacks of the past ten years. As a result, gender-relevant perspectives have increasingly found their way into narratives about right-wing terror. This article examines the contradictory effects of talking about misogyny and right-wing terror in recent debates. The paper traces a continuity of singling out right-wing and misogynist violence as well as an exoneration of the majority society from a shared responsibility for right-wing terror.
\end{abstract}

Keywords Right wing terror - NSU · Antifeminism · Misogyny and hatred of women $\cdot$ Incels

Juliane Lang $(\bowtie)$

Justus-Liebig-Universität Gießen, Gießen, Deutschland

E-Mail: Juliane.lang@ sowi.uni-giessen.de 


\section{Einleitung}

Im Herbst 2021 jährt sich der Jahrestag der Selbstenttarnung des NSU zum zehnten Mal. Nicht nur die Taten als solche stehen für sich, handelt es sich bei den zehn Morden und drei Sprengstoffanschlägen doch um die ,längste und tödlichste Mordserie durch eine neonazistische Vereinigung in Deutschland nach 1945“ (NSUWatch 2020, S. 14). Auch die Aufarbeitung des NSU-Komplexes steht für einen gesellschaftlichen Prozess von zuvor nicht gekannten Dimensionen, dessen Ende noch längst nicht greifbar ist (z. B. Karakayali et al. 2017). Ausgehend vom NSU, dessen Selbstenttarnung das Reden über rechte Gewalt und insbesondere rechten Terror verändert hat, blicke ich auf rechten Terror nach dem NSU. Dieser unterscheidet sich von vorherigen Taten unter anderem durch die Radikalisierung der Täter:innen im Internet sowie die Bedeutung von Geschlecht und insbesondere Männlichkeit in Radikalisierungsprozessen und Tatverläufen. So scheint es zunächst wenig verwunderlich, dass Männlichkeit und der Hass auf Frauen im Reden über die Taten mehr Aufmerksamkeit erfahren. Und dennoch bricht das Reden über rechten Terror nach dem NSU nicht mit anderen gängigen Narrativen der Verbesonderung und Veränderung der Täter:innen und ihrer Taten. Ganz im Gegenteil: So wird unter Rückgriff auf die vergeschlechtlichten Dimensionen rechten Terrors das gern gehegte Narrativ des rechten Terroristen als Sonderling am Rande der Gesellschaft aufrechterhalten. Ausgehend von dieser Analyse argumentiere ich für einen umfassenden Einbezug der Dimension Geschlecht in die Analyse rechten Terrors und gegen eine Verkennung rechter und frauenfeindlicher Gewalt als Phänomen am rechten Rand der Gesellschaft. Denn das Reden über rechten Terror, so meine These, ist auch in den Jahren nach der Selbstenttarnung des NSU paradigmatisch für die Wahrnehmung des Problems.

\section{Der NSU als diskursives Ereignis}

Die Selbstenttarnung des NSU im November 2011 lässt sich als diskursives Ereignis (Jäger 2004, S. 162) lesen: nicht nur, weil es die durch den NSU begangenen Morde und Anschläge in einen Zusammenhang setzte und der Öffentlichkeit gegenüber bekannt machte. Sondern es änderte das Reden über Rechtsextremismus und rechten Terror in der bundesdeutschen Gesellschaft (vgl. Quent 2016, S. 13). Umfängliche Untersuchungen in den auf die Selbstenttarnung folgenden Jahren offenbarten Schritt für Schritt, wie Ermittlungsbehörden, Geheimdienste, Politik und Öffentlichkeit systematisch in der Aufklärung der Taten und der Anerkennung von Angehörigen und Betroffenen versagten. Lautstark meldeten sich Angehörige und Betroffene der rechtsterroristischen Taten zu Wort und forderten ein, was ihnen über die Jahre vorenthalten worden war: Sichtbarkeit und Anerkennung für sich und ihr Wissen um die Botschaften hinter den Taten (z. B. John 2014). Denn auch wenn der NSU bis zur Selbstenttarnung keine Bekennerschreiben hinterließ, handelte es sich um Botschaftstaten: Der rechte Terror hinterließ bewusst gesetzte Botschaften an Angehörige migrantischer Communities in Deutschland, die von diesen gehört wurden. Im Zuge der Aufarbeitung des NSU-Komplexes ließ sich dieses, verschiedent- 
lich als „migrantisch situiert“ beschriebene Wissen (Güleç und Schaffer 2017) nicht länger ignorieren. Dies bedeutete einen in Teilen vollzogenen Bruch mit der vorherigen Ignoranz der Mehrheitsgesellschaft gegenüber den Betroffenen, dem „Nichtsehen-wollen rechten Terrorismus" "(Schmincke und Siri 2013, S. 15). So haben Angehörige, Freund:innen und Unterstützer:innen der Ermordeten seit 2006 auf einen möglichen Zusammenhang der neun Morde an migrantischen Männern und ein rassistisches Motiv hingewiesen. Ihr Wissen wurde ignoriert, sowohl von den Ermittlungsbehörden als auch von der Öffentlichkeit. Ganz im Gegenteil: In der öffentlichen Berichterstattung wurde noch bis ins Jahr 2011 der menschenverachtende Begriff der „Dönermorde“ verwandt (Virchow et al. 2015).

Der Selbstenttarnung und dem Wissen um die Täterschaft des Kerntrios Zschäpe, Mundlos und Böhnhardt folgte eine Reihe an Parlamentarischen Untersuchungsausschüssen. Vieles von dem, was heute bekannt ist, entstammt dem akribischen Aktenstudium von Mitarbeitenden der Untersuchungsausschüsse des Bundestages und insgesamt acht Länderparlamenten. In der Berichterstattung zum NSU-Komplex allgemein und in der Arbeit der Untersuchungsausschüsse im Besonderen wurden zuvor mehrheitlich in Fachkreisen geführte Debatten um Rechtsextremismus und Rechtsterrorismus einer breiten Öffentlichkeit zugänglich gemacht. Journalistische und populärwissenschaftliche Arbeiten beschäftigten sich mit der Vorgeschichte des NSU und dessen Entstehungsgeschichte. Sie warfen einen Blick auf die gesellschaftlichen Bedingungen der 1990er-Jahre, unter denen sich die späteren Täter:innen sozialisierten, politisch radikalisierten und letztlich für den Weg in den (vermeintlichen) Untergrund entschieden. Ein Teil der Arbeiten floss in Fernsehproduktionen wie den von der ARD produzierten Dreiteiler „Mitten in Deutschland: NSU“ ein und fand damit Eingang in die kulturelle Repräsentation rechten Terrors allgemein und des NSU-Komplexes im Besonderen.

Heute, zehn Jahre nach der Selbstenttarnung des NSU, lassen sich zwei grundsätzliche Diskursstränge in der Aufklärung der Taten des NSU beschreiben: Ein sicherheitspolitischer Diskurs(-strang), der mit der sog. Triothese aufrecht zu erhalten suchte, was die gesellschaftliche Wahrnehmung rechten Terrors lange Zeit prägte. Es handelt sich hierbei um die Annahme, die rechten Täter:innen hätten jeweils allein, ohne substantielle Unterstützung von außen gehandelt. Der Gesellschaft kommt keine weitergehende Verantwortung zu, schließlich hätten die Täter:innen im Verborgenen gehandelt und seien in ihrer Klandestinität nicht greifbar gewesen. Die Einzeltätertheorie wurde im Falle des NSU-Komplexes auf das (Kern-)Trio angewandt. Der Generalbundesanwalt stützte die Anklageschrift gegen Beate Zschäpe und (lediglich) vier Mitangeklagte vor dem Oberlandesgericht München maßgeblich auf die These, beim NSU habe es sich um eine abgrenzbare Zelle dreier Personen gehandelt, von denen zwei zum Zeitpunkt der Anklage tot waren (NSU-Watch 2020, S. 81ff.). Die Mitangeklagten wurden für unterschiedliche Unterstützungsleistungen, insbesondere bei der Beschaffung der Mordwaffe und der Bereitstellung von Papieren für das Leben im Untergrund, angeklagt.

Als zweiter, zeitlich parallel verlaufender, Diskursstrang lässt sich der von Betroffenen initiierte und von einer solidarischen Zivilgesellschaft fortgesetzte Diskurs um die Verantwortung von Staat und (Mehrheits-)Gesellschaft im NSU-Komplex ausmachen. In diesem wird nicht nur ein Versagen des Staates konstatiert, sondern 
es werden einzelne gesellschaftliche Teilbereiche und -öffentlichkeiten mit ihrer jeweiligen Verantwortung konfrontiert. Journalistische Arbeiten stellen der pädagogischen Arbeit mit rechtsorientierten Jugendlichen in den 1990er-Jahren kein gutes Zeugnis aus (Kleffner 2015). Die bundesdeutschen Leitmedien werden ihrerseits an ihre rassistische Berichterstattung zu den sog. Ceska-Morden erinnert (Virchow, Thomas, und Grittmann 2015). Der NSU wird als „Komplex“ beschrieben, um weg von der Verantwortung dreier Einzelpersonen und hin zur Verantwortung der Gesellschaft für die Taten und deren (Nicht-)Aufklärung zu kommen. Auch die vielfältigen Unterstützungsleistungen, die das NSU-Kerntrio wissentlich und unwissentlich von rechten Szeneangehörigen und anderen Personen erhalten hat, werden hier mit benannt und finden beispielsweise Eingang in eine symbolische ,gesellschaftliche Anklage des Rassismus“ (Lückenlos e. V. 2020).

In der juristischen Bewertung des NSU-Komplexes hat sich bislang die sog. Triothese durchgesetzt, mit Folgen auch für die gesellschaftliche Debatte um rechten Terror allgemein und den NSU-Komplex im Besonderen. Der sicherheitspolitische Diskursstrang kann sich bislang als hegemonial behaupten. Und dennoch ist der zivilgesellschaftliche Diskursstrang damit nicht verstummt. Beide Diskursstränge - der sicherheitspolitische wie der zivilgesellschaftliche - finden sich heute im Reden über rechtsterroristische Taten nach dem NSU wieder und streiten um Deutungsmacht. Insbesondere der sicherheitspolitische Diskurs, in dessen Zentrum die Betrachtung rechten Terrors als Taten gesellschaftlich isolierter Einzeltäter:innen steht, erweist sich dabei als erstaunlich wandlungsfähig, auch im Blick auf die Veränderung rechten Terrors und neue Tätertypen.

\section{Rechter Terror nach dem NSU: Einzeltäter aus dem Netz?}

Auch wenn die Taten des NSU als singulär in der deutschen Geschichte zu betrachten sind, auch weil es den Täter:innen gelang, über eine Zeitspanne von fast 14 Jahren unerkannt zu morden, sind sie dennoch nicht das einzige Beispiel rechten Terrors in Deutschland. Der Politikwissenschaftler Fabian Virchow erinnert in einem der zunächst wenigen wissenschaftlichen Sammelbände nach der Selbstenttarnung des NSU an die Kontinuität rechten Terrors in der Bundesrepublik: Er verweist auf Kontinuitäten und Unterschiede zum bis dato bekannten Rechtsterrorismus der 1970er und 1980er-Jahre und spricht vom NSU als „Prisma“ in Bezug auf rechten Terror(ismus) in Deutschland (Virchow 2013).

Die Morde und Anschläge des NSU blieben auch nicht die letzten rechtsterroristischen Taten in Deutschland, es folgten weitere Anschläge nach 2011: Am 22. Juli 2016 schoss der 18-jährige Deutsch-Iraner David Sonboly auf Jugendliche mit Migrationsgeschichte an einem Münchner Einkaufszentrum. Neun Jugendliche verloren ihr Leben, der Täter erschoss sich später selbst. Er wählte für seine Taten den Jahrestag des rechtsterroristischen Anschlags von Anders Breivik im norwegischen Oslo sowie auf der Insel Utoya. ${ }^{1}$ Am 9. Oktober 2019 versuchte ein Mitte

\footnotetext{
1 Zur politischen Bewertung des Attentates am Münchner Olympia-Einkaufszentrum siehe die von der Stadt München in Auftrag gegebenen Gutachten: Hartleb (2017); Kopke (2017); Quent (2017).
} 
20-jähriger Täter sich am höchsten jüdischen Feiertag Zutritt zur Synagoge in Halle zu verschaffen; nachdem dies misslang, tötete er vermeintlich willkürlich zwei Menschen und verletzte weitere schwer. Der Täter filmte sich bei der Tat selbst, die von ihm in die Kamera gesprochenen Worte sind voller Menschenfeindlichkeit und Zynismus. Kein halbes Jahr später, am 22. Februar 2020, erschoss ein Mitte 40-jähriger Mann in der hessischen Stadt Hanau neun Menschen mit Migrationsgeschichte. Er verletzte weitere Menschen schwer und erschoss später zunächst seine Mutter und dann sich selbst. Auch er hinterließ Dokumente, aus denen seine zutiefst rassistischen Überzeugungen und seine Menschenfeindlichkeit offen hervorgehen.

Die Journalistin Andrea Röpke spricht in Bezug auf den Attentäter vom Münchner Olympia-Einkaufszentrum von einem ,Typus neuer Täter, die vornehmlich online unterwegs und vernetzt sind“ (Röpke 2020, S. 109). Beides trifft auch auf die Täter von Halle und Hanau zu. Wenn auch unterschiedlichen Alters, handelt es sich in allen Fällen um Männer, die sich im Internet vernetzt und ihre Anschlagspläne digital weiterentwickelt und verfestigt haben. Der Attentäter von München stand trotz seines jungen Alters im Austausch mit us-amerikanischen Rechtsextremen. ${ }^{2}$ Der Attentäter von Halle kündigte seine Tat kurz vorher im Netz an und streamte sich selbst mittels einer Helmkamera auf einer Plattform, die vor allem in GamerKreisen für die Übertragung von Videospielen in Echtzeit genutzt wird. Analog zu einem Videospiel konnten ihm Zuschauer:innen weltweit und live während der Morde und seiner anschließenden Flucht folgen. Dies war Anlass dafür, dass im Nachgang verschiedentlich von einer Gamification des rechten Terrors die Rede war. In der sozialwissenschaftlichen Rechtsextremismusforschung wird die Verwendung des Begriffs kritisiert, weil dies einen Übertrag der Logiken des Videospielens auf rechten Terror bedeute. Und mehr noch:

Die Rede von der „Gamifizierung des Terrors“ impliziert einen grundlegenden Wandel rechtsterroristischer Gewalt. Dies ist aber nicht zutreffend. Zwar tritt global ein neuer Tätertypus auf, der stärker als andere Täter*innen durch radikale Online-Milieus, wie sie sich auf 8chan und anderswo finden, geprägt ist und diese zu wichtigen Adressat*innen auserkoren hat. Dieser neue Tätertypus hat aber nur einen sehr kleinen Anteil an den schweren Gewalttaten mit rechter Motivation. (Puls 2020, S. 7)

Eine Wahrnehmung von rechtem Terror als Taten einzelner, der Gamer-Szene nahestehenden Täter:innen lenke damit ab von all jenen, die dem Muster nicht entsprechen - und weiterhin Anschläge, etwa auf Unterkünfte geflüchteter Menschen, verüben. Viel wurde seitdem gesprochen über die Radikalisierung jener Attentäter, die als Lone-Wolf - als einsame Wölfe - charakterisiert werden. Dabei steht

[d]ie Bezeichnung „Einzeltäter“ ... in diesen Fällen lediglich für die konkrete Tatplanung. Sie verneint nicht, dass die einschlägige Gewalt- und Ideologiefixierung der Täter Ursachen hat, dass ihre Taten Folge von Kommunikation

\footnotetext{
2 Einer von diesen wurde später selbst zum Täter und erschoss im Dezember 2017 zwei Jugendliche mexikanischer Herkunft und anschließend sich selbst in einer Schule in New Mexico, USA (z. B. Hartleb 2020).
} 
und Interaktion mit Gleichgesinnten sein können und dass die Akteure sich angesichts von zunehmender Fremdenfeindlichkeit in der Gesellschaft und des damit einhergehenden Diskurses motiviert fühlen. (Hartleb 2020, S. 12)

Was in der Aufzählung fehlt sind ein gewaltvoller Frauenhass und ein mitunter offener Antifeminismus, der sich in gesellschaftlichen Debatten der vergangenen Jahre zeigte und an den die Täter:innen anschließen konnten (Autor*innenkollektiv Fe.In 2019).

Wenn heute der Tätertypus des „Einsamen Wolfes“ als in Gänze neues und dem Internet entsprungenes Phänomen beschrieben wird, so trifft dies nur in Teilen zu. Rechtsterroristische Anschläge wurden seit Jahren als Taten Einzelner verkannt: Ein Beispiel ist der Anschlag auf das Münchner Oktoberfest im September 1980. Journalist:innen äußerten schon früh Zweifel an der offiziellen Geschichtsschreibung, nach der es sich beim bis dato größten Anschlag in der Geschichte der Bundesrepublik um die Tat eines verwirrten Einzeltäters handle. Journalist:innen sammelten in den Folgejahren Anhaltspunkte dafür, dass der Täter sich im Umfeld der verbotenen Wehrsportgruppe Hoffmann bewegte. Wir wissen somit heute, dass auch der Täter des Oktoberfestattentates im Austausch mit Gleichgesinnten stand, die ihn vermutlich in seinen Plänen bestärkt haben - eine konkrete Tatbeteiligung konnte bis heute niemandem nachgewiesen werden. Neu ist dagegen sehr wohl, dass jener Austausch in Echtzeit mit Gleichgesinnten in aller Welt stattfinden kann. Und somit ist davon auszugehen, dass u. a. die Schriften des us-amerikanischen Rechtsextremen Jack Donovan bereits vor seiner Veröffentlichung im deutschsprachigen Antaios-Verlag ein deutsches Publikum fanden: Donovan bringt darin in besonders perfider Weise einen ausgeprägten Männlichkeitskult zusammen mit der Aufforderung, ,,sich zur Wehr zu setzen" gegen alles, was die uneingeschränkte Vormachtstellung des weißen Mannes in Frage stellt (vgl. Volpers 2020).

\section{Geschlecht und Geschlechterverhältnisse im Rechtsterror}

Die offen zur Schau gestellte Misogynie, ihr ausgeprägter Antifeminismus und ihr widersprüchlicher Bezug auf eine männliche Vormachtstellung unterscheidet die jüngeren rechtsterroristischen Täter von früheren Tätern. Der NSU ermordete aus vordergründig rassistischen Motiven neun migrantische Männer. Aufzeichnungen, die am letzten Wohnort des Kerntrios gefunden wurden, zeugen bereits im NSUKomplex von der Bedeutung der Kategorie Geschlecht, u. a. für die Auswahl der Opfer: So berichten Journalist:innen, dass sich Mundlos, Böhnhardt und Zschäpe bei der Auswahl ihrer Opfer auf ,unarische“ Männer im zeugungsfähigen Alter konzentrierten. ${ }^{3}$ In einem Fall wurde von der Ermordung eines türkischen Unterneh-

\footnotetext{
3 Auch spielten Geschlecht und vor allem Geschlechterverhältnisse eine Rolle sowohl im Binnenverhältnis des Kerntrios als auch nach außen: Beate Zschäpe befand sich vor dem gemeinsamen Abtauchen nacheinander in Beziehungen zu beiden Männern. Nach dem Abtauchen stellten sich Zschäpe, Mundlos und Böhnhardt Nachbar:innen gegenüber als Liebespaar mit einem Bruder des Mannes vor. Sie nutzten damit klassische heteronormative Erzählungen als Legende, um keine Aufmerksamkeit für ihr Zusammenleben zu wecken (vgl. Köttig 2013).
} 
mers in Dortmund abgesehen, weil er über 60 Jahre und damit zu alt war, um ins Raster des Kerntrios zu fallen. Dahinter verbirgt sich die extrem rechte und rassistische Erzählung vom bedrohlichen fremden Mann - eine Erzählung, die einige Jahre nach der Selbstenttarnung des NSU zum Kernbestandteil breiterer gesellschaftlicher Debatten rund um die Vorfälle in der Silvesternacht in Köln wurde (Madjlessi-Roudi 2018). Sie findet sich zugespitzt in der Erzählung vom „Großen Austausch“, mit dem die zeitgenössische autoritäre und extreme Rechte in den vergangenen Jahren ihre Dystopie vom Austausch der Deutschen durch andere „Völker“ begründet (Goetz 2020).

Und sie findet sich so eins zu eins beim Täter von Halle, der seinen Hass auf Frauen unter anderem damit begründet, dass diese zu wenige - oder gar die falschen Kinder bekommen würden und damit die Zukunft des deutschen Volkes ins Ungewisse falle. Während der NSU migrantische Männer ermordete, um zu verhindern, dass diese Kinder in Deutschland zeugen, macht der Täter von Halle explizit Frauen und Feministinnen verantwortlich für einen angeblichen Niedergang des deutschen Volkes. Die dahinterstehende Vorstellung von Gesellschaft ist dieselbe: Weiße deutsche Frauen sollen weiße deutsche Kinder von weißen deutschen Männern bekommen und damit den Fortbestand der Volksgemeinschaft sichern. Eine Erzählung, die im deutschen Kolonialismus ihren Ausgang nahm und noch mehr als ein Jahrhundert später zentraler Bestandteil von Bevölkerungspolitiken der autoritären und extremen Rechten ist (vgl. Botsch und Kopke 2018; Goetz 2020), und die den deutschen Männern im rechten Denken die Rolle des Kriegers zuweist. Der Soziologe und Männlichkeitsforscher Michael Meuser stellt heraus, wie neben dem zu einem bestimmten historischen Zeitpunkt gültigen hegemonialen Männlichkeitsbild nach Connell (2015) weitere Bilder existieren können, die für ein jeweils spezifisches Milieu Hegemonialität entfalten (Meuser 2010). In der Rechtsextremismusforschung wird dies auf den Forschungsgegenstand angewandt: Während die Figur des ,soldatischen Mannes“ nach 1945 gesellschaftlich an Hegemonie verlor, blieb sie hegemonial in Männlichkeitsbildern der autoritären und extremen Rechten (z.B. Virchow 2010) - und erfährt im rechten Terror aktuell eine Revitalisierung.

Denn gerade bei den männlichen, allein handelnden Attentätern von München, Halle und Hanau ist die offensichtliche Inszenierung von Männlichkeit als Begleitmusik der Taten zu betrachten: wenn auch nicht als alleiniges Erklärungs-, so doch als tatunterstützendes Motiv. Zwei der drei Täter thematisierten offen Geschlecht und Geschlechterverhältnisse in ihren Ausführungen: Der Täter von Halle formulierte in einer Deutlichkeit, wie vor ihm andere rechte Attentäter wie Anders Breivik, eine Erzählung vom gesellschaftlichen Verfall aufgrund von Geschlechterverhältnissen, die in Unordnung geraten seien. Er machte „den Feminismus“ verantwortlich für sein eigenes Unglück und die gesellschaftliche Lage Deutschlands. In seiner Vernehmung gibt er zu Protokoll: „Männer wie ich bekommen auch deshalb keine Frau, weil Ausländer sie uns wegnehmen“ (zitiert nach: Gesellmann 2021). Auch der Täter von Hanau spricht in seinem Pamphlet über sein Scheitern in der Suche nach einer (heterosexuellen) Beziehung. Er spricht außerdem über die Rolle seiner Mutter in seinem Leben und erschießt diese, bevor er sich selbst das Leben nimmt. Was sich hier als Muster zeigt, beschreibt der Soziologe und Männlichkeitsforscher Michael Kimmel als ,aggrieved entitlement“ (Kimmel 2013): ein ,gekränktes An- 
spruchsdenken“, aufgrund dessen sich weiße Männer nehmen würden, was ihnen aus ihrer Sicht zustehe - und sich selbst den Ort in der Geschlechterordnung zuweisen, der ihnen vermeintlich zustehe. Im rechten Terror verknüpft sich ein autoritär und extrem rechtes Denken mit der Geringschätzung von Frauen und jener gekränkten Anspruchshaltung, die Kimmel beschreibt (siehe auch: Kaiser 2020, S. 108). In der Figur des Lone Wolfs kommt all dies zusammen: die Figur steht stellvertretend sowohl für das Streben nach hegemonial-soldatischer Männlichkeit - und dem Scheitern daran.

Geschlecht und Geschlechterverhältnisse waren somit nie nur ein Nebenschauplatz im rechten Terror, sondern prägten diesen ideologisch wie auch auf der Akteursebene. Dennoch spielten dessen vergeschlechtlichte Elemente lange Zeit eine untergeordnete Rolle in gesellschaftlichen Narrativen über rechten Terror. Dies veränderte sich im Hinblick auf die rechtsterroristischen Anschläge nach dem NSU.

\section{Kontinuitäten der Einzeltäterthese}

Eine in Deutschland noch junge Debatte ist die über die (Selbst-)Radikalisierung von Incels (,,involuntary celibat") - jenen ,unfreiwillig zölibatär“ lebenden Männern vorwiegend jüngeren Alters. Ein Phänomen, welches in den USA schon länger Thema ist (vgl. Manne 2019), erfährt mit den vordergründig rassistischen und antisemitischen Anschlägen von Halle und Hanau auch in Deutschland eine gestiegene Aufmerksamkeit. Christina Feist, Überlebende des Anschlags von Halle, benennt deutlich die Frauenfeindlichkeit des Täters in ihrem Schlussplädoyer als Nebenklägerin im Prozess gegen den Täter vor dem Landgericht Magdeburg (Feist 2020). Auch wenn darauf verwiesen wird, dass es sich beim Attentäter von Halle nicht um einen klassischen Incel handelte, entstand eine öffentliche Debatte um die Dimensionen von Frauenfeindlichkeit und offener Misogynie im rechten Terror und in den Jugendzimmern heranwachsender junger Männer. Anders als zu vermuten wäre, stellt das neuerliche Interesse an den vergeschlechtlichten Dimensionen rechten Terrors andere gängige Narrative im Reden über rechten Terror dabei nicht in Frage. Ganz im Gegenteil: Das Reden über rechten Terror nach dem NSU bedient sich gängiger Narrative des sicherheitspolitischen Diskurses und restabilisiert diesen im Rückgriff auf die vergeschlechtlichten Dimensionen jüngerer rechtsterroristischer Attentate.

Ein Beispiel: Im März 2021, ein gutes Jahr nach den Anschlägen von Hanau und etwa anderthalb Jahre nach dem Anschlag in Halle, beschäftigte sich eine öffentlich-rechtliche Fernsehproduktion mit den Themen Incels, Gewalt gegen Frauen und rechter Terror. Unter dem Titel „Borowski und die Angst der weißen Männer“ ermittelte ein „Tatort“ im Themenkomplex rechter Terror und Incels, und bereitete das Thema damit für ein Millionenpublikum auf (Weegmann 2021). Verschiedentlich als „Archiv der Gegenwartsgeschichte“ (Wenzel 2000) beschrieben, bildet der „Tatort" nicht einfach Themen ab, sondern misst diesen gesellschaftliche Relevanz zu. Die Tatortreihe

fungiert dabei sowohl als eine Art Brennspiegel, der Themen in den gesell- 
schaftlichen Fokus rückt, als auch als eine Art Zerrspiegel - aber nie als neutrale Beobachterin. Sie bildet bundesrepublikanische Lebensverhältnisse nicht $\mathrm{ab}$, sondern konstruiert Vorstellungen, die man als deren Abbilder akzeptieren kann. (Hißnauer, Scherer, und Stockinger 2014, S. 12)

Das Thema Incels und rechte Gewalt zum Inhalt eines „Tatort“-Krimis zu machen, sagt somit etwas aus über die Relevanz des Themas Geschlecht und frauenfeindliche Gewalt im aktuellen gesellschaftlichen Reden über rechten Terror. Der Anschlag von Halle erscheint als Schablone des jungen männlichen Protagonisten im „Tatort“. Im Film beobachten die Zuschauer:innen einen jungen Mann, wie er zunächst bei zaghaften Flirtversuchen mit einer jungen Frau scheitert und anschließend von einer autoritären Vorgesetzten in seinem prekären Job als Parkwächter abschätzig behandelt wird. Seine aufkommende Enttäuschung über den Verlust der potenziellen Beziehungspartnerin und die Wut auf sein Umfeld verstärkt sich durch den Konsum von zunächst Pick-Up- ${ }^{4}$ und später Incel-Foren im Netz. Er wird im Laufe der 90 min zum potenziellen Mörder, ein größerer Anschlag kann nur durch das schnelle Handeln der Kommissar:innen verhindert werden.

Ein anderes Beispiel: In einer öffentlich-rechtlichen TV-Dokumentation aus dem August 2020 Jahres schauen die Zuschauer:innen dem sich radikalisierenden jungen Mann regelrecht über die Schulter: Der (potenzielle) Täter befindet sich in einem digital nachgestellten Jugendzimmer, bestehend aus Bett und PC-Ecke (vgl. Bergmann und Barth 2020). Das Setting ist paradigmatisch, und verrät viel über die Bilder, welche der gesellschaftlichen (Nicht-)Wahrnehmung von rechtem und frauenfeindlichen Terror zugrunde liegen. Ein zunächst unauffälliges Verhalten im Alltag kennzeichnet die Figur der Protagonisten in beiden Produktionen. Der Täter wird jeweils porträtiert als sozial weitestgehend zurückgezogen, und dennoch online gut vernetzt. Anders als in anderen TV-Produktionen über Incels, in denen die sich radikalisierenden Männer unabhängig vom tatsächlichen Alter im Jugendzimmer des elterlichen Hauses gezeigt werden, bewohnt der Protagonist im ,Tatort“ seine eigene Wohnung. Und dennoch entspricht auch diese Wohnung dem Klischee eines jungen Mannes; im Mittelpunkt seines Lebens wie auch seiner Wohnung steht der Computer mit dem Zugang ins sog. Darknet, jenen versteckten Teilen des Internets, die nicht auf den ersten Klick im Standard-Browser zu finden sind.

Bilder, die hier und anderenorts zur Charakterisierung des typischen Incels verwandt werden, beschreiben ganz normale junge Männer, die daran scheitern, Beziehungen zu Frauen aufzubauen - und mit jeder Zurückweisung oder jedem missglückten Versuch der Kontaktaufnahme weiter verzweifeln. Sie scheitern zugleich am Wunsch und an dem sich selbst gesteckten Anspruch, eine heterosexuelle Beziehung einzugehen - und irgendwann wendet sich ihre Enttäuschung in einen Hass

\footnotetext{
4 Bei Pick-Up-Artists handelt es sich um Gruppen von Männern, die sich in Internetforen sowie ,in Seminaren und gemeinsamen Clubbesuchen organisieren, um die ,Kunst' zu erlernen und weiterzuentwickeln, wie man möglichst viele Frauen abschleppt“ (Kaiser 2020, S. 36). Pick-Up-Artists werden als Teil der so genannten Mannosphäre (,Manosphere“) im Internet beschrieben. Nagle und andere beschreiben damit nicht ,„,die Männerbewegung ' an sich ... sondern ... ihre finstere Schattenseite, die sich im Internet entfaltet hat" (Nagle 2018, S. 106).
} 
auf Frauen und ein übersteigertes Anspruchsdenken, welches Kimmel (2013) als Motiv der misogynen Gewalt ausmacht.

Die Metapher des Jugendzimmers im elterlichen Keller oder des zurückgezogenen Einzelgängers erwecken zugleich den Eindruck, jene Männer radikalisierten sich im Verborgenen, ja gar in einer Art Untergrund, unentdeckt vom Rest der Gesellschaft. Und hier bedient sich das Reden über Incels und rechten Terror nach dem NSU an Versatzstücken eines sicherheitspolitischen Diskurses um rechte Terroristen, die sich unentdeckt vom Rest der Gesellschaft radikalisieren, zur Tat schreiten und ohne das Wissen oder gar Zutun anderer zu Einzeltätern werden. Die Täter werden diskursiv außerhalb der Gesellschaft oder an ihren extremen Rändern verortet. Dabei befinden sich die ,verborgenen“ Orte der Radikalisierung potenziell in jedem Reihenhaus mit ausgebautem Keller, was die Unmittelbarkeit der Bedrohung durch Incels umso größer macht. Auch wenn Kracher betont, dass es sich bei Incels de facto eben nicht um Einzeltäter handele (Kracher 2020a, S. 80), trifft für den Diskurs über sie zu, was Hartleb für den Tätertypus des ,Einsamen Wolfs“ schreibt:

So herrscht die Ansicht vor, es handele sich einfach um höchst merkwürdige Personen, denen ,nicht mehr zu helfen war“, da sie die ausgestreckte Hand im familiären und sozialen Umfeld nicht ergriffen hätten. Gegen gestörte Einzeltäter ist man eben machtlos, heißt es oft. Wie einst der legendäre Kaspar Hauser scheinen sie einfach aus einer anderen Welt zu stammen, kaum ergründbar für Bürger und Polizei. Doch eine solche Betrachtung spricht die Gesellschaft von jeder Schuld frei - mit fatalen Folgen für die Opfer, deren Angehörige, die Strafverfolgung und die Prävention. (Hartleb 2020, S. 100)

Auch in der Rede davon, jene Täter seien ,kaum ergründbar für Bürger und Polizei“, zeigen sich Parallelen zum NSU-Komplex: So nutzten diverse Vertreter:innen insbesondere beteiligter Ermittlungsbehörden dies als Schutzbehauptungen, um von den systematischen Fehlern der eigenen Ermittlungsarbeiten abzulenken und den rechten Terror und mit ihm die rechten Terrorist:innen als weit weg an den Rändern der Gesellschaft zu verorten. Das Reden über Incels als „Sonderlinge“ wiederholt dieses Muster: Es verortet sie weit weg an den Rändern der Gesellschaft. Es verbesondert sie und entlastet die Mehrheitsgesellschaft von der Verantwortung für die Radikalisierung rechter Täter in ihrer Mitte, ähnlich wie Hartleb es für den Tätertypus des „Einsamen Wolfes“ beschreibt. In einem Kommentar des Redaktionsnetzwerks Deutschland wird wenige Tage nach dem Anschlag in Hanau von einer „Integrationsaufgabe ganz unerwarteter Art“ in Bezug auf Incels gesprochen (Koch 2020), und damit ausgedrückt, diese stünden außerhalb der (Mehrheits-)Gesellschaft. Florian Hartleb beschreibt dieses Muster als ,psychologischen Trick“, der darin bestehe, ,den Akteur als „Kranken“ und „Fremden“ darzustellen. Zu schmerzhaft ist der Befund, dass der Terrorismus unter uns entsteht, im stillen Kämmerlein gedeiht und dann explosiv seine Wirkung entfaltet“ (Hartleb 2020, S. 44). 


\section{Im Fokus: Männlichkeit}

Neuere Arbeiten zu rechtem und frauenfeindlichem Terror dagegen wählen einen anderen Zugang in ihrer Analyse des Phänomens - und bewegen sich damit bewusst und unbewusst in der Tradition jenes Diskursstranges, der mit einer umfassenden Perspektive auf rechten Terror als „Komplex“ blickt. Sie begreifen Incels nicht als Phänomen außerhalb, an den Rändern der Gesellschaft, sondern als Ausdruck gesellschaftlicher Geschlechterverhältnisse.

Incels sollten ... nicht als ... Ausnahme des gesellschaftlichen Geschlechterverhältnisses gesehen werden, sondern ihre extreme Ideologie muss als Spitze des Eisbergs verstanden und erklärt werden, der sich aus männlichem Anspruchsdenken, der narzisstischen Kränkung, dieses nicht erfüllt zu bekommen, und gesellschaftlich vermittelter Frauenfeindschaft zusammensetzt. (Kracher 2020a, S. 79)

Das Problem sind nicht die gescheiterte Männlichkeit, die falschen Männlichkeitsbilder oder das übermäßige Streben nach Männlichkeit durch die späteren Täter. Das Problem ist Männlichkeit als Strukturprinzip moderner Gesellschaften, welches Jungen und Männer scheitern lässt. Der Blick auf Männlichkeit als Strukturprinzip von Gesellschaft und die paradoxen Effekte der Aneignung und des Scheiterns daran entlastet weder die Täter noch die Gesellschaft von einer individuellen oder kollektiven Verantwortung: Aber es widerspricht der gern gehegten Vorstellung, die Täter seien sonderliche „Einzelgänger [die] zu rechten Attentätern“ werden, wie es die zitierte TV-Dokumentation behauptet (Bergmann und Barth 2020).

Eine solche Perspektivverschiebung - weg von den Rändern der Gesellschaft und den ,,verborgenen Strukturen“ im Untergrund, hin zu den Orten ihrer Radikalisierung innerhalb gesellschaftlicher (Geschlechter-)Verhältnisse - zeigt gleichzeitig die Grenzen der Wahrnehmung rechter und frauenfeindlicher Terroristen als ,Sonderlinge" auf. Denn wenn wir in der Analyse davon ausgehen, dass es sich bei einem Phänomen wie der Incel-Bewegung um einen im besten Sinne des Wortes ,homemade terrorism" handelt, dann funktionieren die Prinzipien von Verbesonderung und Veranderung rechten Terrors und rechter Terrorist:innen nicht mehr. Und wo dies nicht mehr gelingt, gerät auch die damit intendierte Entlastung der Mehrheitsgesellschaft aus der Verantwortung an ihre Grenzen.

Umso mehr gerät in den Blick, wie versucht wird, die Wahrnehmung rechten Terrors als Phänomen außerhalb oder im Verborgenen der Gesellschaft regelrecht krampfhaft aufrecht zu erhalten. Beispiele hierfür finden sich in der medialen Repräsentation rechten Terrors ebenso wie in fach- und populär-wissenschaftlichen Arbeiten zum Thema. Dort wird Antifeminismus und Frauenhass als ,die Einstiegsdroge“ in den Rechtsextremismus oder gar Rechtsterrorismus beschrieben (vgl. Höppner 2020; Kracher 2020b), und damit diskursiv herausgetrennt aus der gesellschaftlichen Normalität. Dabei sind die Grundbestandteile der Incel-Ideologie - die Abwertung von Frauen bis hin zu frauenfeindlicher Gewalt - fester Bestandteil gesellschaftlicher Geschlechterverhältnisse. Präziser wäre es deswegen, von antifeministischen 
und frauenfeindlichen Einstellungen als ideologischen Türöffnern zu sprechen ${ }^{5}-$ und damit aufzuzeigen, welche Gefahr davon ausgeht, wenn sich die Abwertung von Frauen* (und Queers) mit weiteren Elementen extrem rechter Ideologie mischt. Antifeminismus ist fester Bestandteil autoritär und extrem rechten Denkens (Lang 2015) - und verfügt dennoch über eine stabile Verankerung in allen Teilen der Gesellschaft (Höcker et al. 2020). Nicht jeder Incel entwickelt sich zum überzeugten Neonazi oder gar Rechtsterroristen. Und dennoch verdient die widersprüchliche Verbindung von frauenfeindlichem Denken und anderen Teilen autoritär und extrem rechten Denkens eine besondere Aufmerksamkeit, insbesondere in Bezug auf die sich gegenseitig verstärkende Wirkung - wie sie sich im krassesten Fall in rechtem Terror ausdrückt.

\section{Gewalt gegen Frauen}

Ein sicherheitspolitischer Blick auf rechten Terror, der die Täter:innen und ihre Taten an den Rändern der Gesellschaft zu verorten sucht, vermag es nicht, die Komplexität rechten Terrors zu erfassen. Und er zeitigt Effekte, sowohl hinsichtlich der Wahrnehmung des Phänomens an sich, als auch in Bezug auf die Wahrnehmung der dem zu Grunde liegenden Motive. Und somit ist ,[d]ie Art, wie wir über Incels sprechen, ... paradigmatisch" noch in anderer Hinsicht, und zwar in Bezug auf die Wahrnehmung von Gewalt gegen Frauen: „Es wurde eine Gruppe von Extremisten identifiziert, die alle wahnhaft sind. ... [es] wird immer wieder versucht, das Phänomen Frauenhass gewissermaßen auszulagern und es zu einem Extremistenphänomen zu machen.“ (Kaiser 2021).

Frauenfeindliches Denken bis hin zum Frauenhass findet seinen Ausdruck im tagtäglichen Umgang mit Frauen in der Öffentlichkeit wie auch im Privaten, in der Nicht-Anerkennung von Gewalt gegen Frauen ebenso wie im Schweigen über Feminizide - solange sie von „ganz normalen Männern“ ausgehen. Die Verbesonderung rechten Terrors gegen Frauen schützt damit auch eine gesellschaftliche Normalität, die den Hass auf und die Abwertung von Frauen nach wie vor nicht überwunden hat.

Eine Stärke des oben erwähnten „Tatorts“ vom März 2021 ist es, neben dem übersteigerten Frauenhass des späteren Täters auch die ganz normale Abwertung von Frauen im Alltag zu zeigen: So werden Erfahrungen der weiblichen Ermittlerin mit frauenfeindlichen Vorgesetzten und Kollegen zum Thema gemacht. Dies ist nicht selbstverständlich. Wie eine Studie im Auftrag der Otto-Brenner-Stiftung herausstellt, wird über die alltäglichsten Formen der Gewalt gegen Frauen nur selten medial berichtet. Im Mittelpunkt der Berichterstattung großer Medien stehen Taten, die mit dem Tod des Opfers enden. Solche Taten machen jedoch nur einen Bruchteil der tatsächlichen Gewalt gegen Frauen aus (Meltzer 2021, S. 2). Die Rede von Einzeltätern findet sich somit auch in der medialen Darstellung von Gewalt gegen Frauen als Einzelfällen (ebd.) wieder - und stützt diese.

\footnotetext{
5 Ich danke Rebekka Blum für die Anregung, vom Antifeminismus und der Abwertung von Frauen als „Türöffnerideologie“ in die autoritäre und extreme Rechte zu sprechen.
} 
Bei allem, was ein kritischer, häufig zivilgesellschaftlicher Diskursstrang der hegemonialen Erzählung rechten Terrors als Handeln einzelner Täter:innen an den Rändern der Gesellschaft entgegen setzen konnte, hat er die Debatten zwar pluralisiert, aber die Diskurshoheit dennoch nicht für sich entschieden. Ganz im Gegenteil: Dem Reden über rechten Terror nach dem NSU ist ein deutliches Interesse zu entnehmen, die Mär vom Einzeltäter [sic], der im Verborgenen handelt, aufrecht zu erhalten. Die neue Debatte um Frauenhass und rechten Terror dient hier als Einfallstor, überwunden geglaubte Narrative zu revitalisieren. Dies birgt heute wie damals Gefahren für eine umfassende Wahrnehmung des Problems. Ein analytischer Zugang, der sich in der Tradition des zivilgesellschaftlichen Diskursstranges im Blick auf den NSU-Komplex versteht, verschiebt den Fokus weg von den Täter:innen, hin $\mathrm{zu}$ den Betroffenen und den Entstehungsbedingungen rechten und frauenfeindlichen Terrors. Hierfür braucht es weitere Forschung, die sich dem komplexen Wechselverhältnis von Rechtsterrorismus und Gesellschaft aus einer geschlechterreflektierenden und vor allem männlichkeitstheoretischen Perspektive annimmt.

Funding Open Access funding enabled and organized by Projekt DEAL.

Open Access Dieser Artikel wird unter der Creative Commons Namensnennung 4.0 International Lizenz veröffentlicht, welche die Nutzung, Vervielfältigung, Bearbeitung, Verbreitung und Wiedergabe in jeglichem Medium und Format erlaubt, sofern Sie den/die ursprünglichen Autor(en) und die Quelle ordnungsgemäß nennen, einen Link zur Creative Commons Lizenz beifügen und angeben, ob Änderungen vorgenommen wurden.

Die in diesem Artikel enthaltenen Bilder und sonstiges Drittmaterial unterliegen ebenfalls der genannten Creative Commons Lizenz, sofern sich aus der Abbildungslegende nichts anderes ergibt. Sofern das betreffende Material nicht unter der genannten Creative Commons Lizenz steht und die betreffende Handlung nicht nach gesetzlichen Vorschriften erlaubt ist, ist für die oben aufgeführten Weiterverwendungen des Materials die Einwilligung des jeweiligen Rechteinhabers einzuholen.

Weitere Details zur Lizenz entnehmen Sie bitte der Lizenzinformation auf http://creativecommons.org/ licenses/by/4.0/deed.de.

\section{Literatur}

Autor*innenkollektiv Fe.In. 2019. Frauen*rechte und Frauen*hass. Berlin: Verbrecher-Verlag.

Bergmann, Christian, und Florian Barth. 2020. Der Terror der einsamen Wölfe: Wie Einzelgänger zu rechten Attentätern werden. MDR.

Botsch, Gideon, und Christoph Kopke. 2018. Der „Volkstod“. Zur Kontinuität einer extrem rechten Paranoia. In Antifeminismus in Bewegung. Aktuelle Debatten um Geschlecht und sexuelle Vielfalt, Hrsg. Juliane Lang, und Ulrich Peters, 63-90. Hamburg: Marta Press.

Connell, Raewyn. 2015. Der gemachte Mann. Konstruktion und Krise von Männlichkeiten, 4. Aufl., Wiesbaden: Springer VS.

Feist, Christina. 2020. „Deutschland hat ein Antisemitismus- und Rassismusproblem.“ Schlusswort von Christina Feist. https://democ.de/artikel/schlusswort-von-christina-feist/. Zugegriffen: 30. Sept. 2021.

Gesellmann, Christian. 2021. Warum ich als Mann über Feminismus schreibe. Krautreporter, 9. April 2021. https://krautreporter.de/3780-warum-ich-als-mann-uber-feminismus-schreibe?shared= 9df7086d-cb5d-471d-b792-8836b5fed395. Zugegriffen: 30. Sept. 2021.

Goetz, Judith. 2020. ,Der große Austausch“ - Rechtsextreme Reproduktions- und Bevölkerungspolitiken am Beispiel der Identitären. Femina Politica 2020(2):37-49. 
Güleç, Ayse, und Johanna Schaffer. 2017. Empathie, Ignoranz und migrantisch situiertes Wissen. Gemeinsam an der Auflösung des NSU-Komplexes arbeiten. In Den NSU-Komplex analysieren, Hrsg. Juliane Karakayali, Çagri Kahveci, Doris Liebscher, und Carl Melchers, 57-80. Bielefeld: transcript.

Hartleb, Florian. 2017. Rechtsextremistisch motivierter Einsamer-Wolf-Terrorismus statt Amoklauf. Eine notwendige Neubewertung der Morde am Olympiaeinkaufszentrum München. https://www. muenchen.de/rathaus/dam/jcr:e26efa5a-1881-4bd6-a417-da264f7e223b/2017-10-06\%20Gutachten $\% 20$ Hintergr\%C3\%BCnde\%20und\%20Folgen\%20des\%20OEZ-Attentats\%20Hartleb.pdf. Zugegriffen: 30. September 2021.

Hartleb, Florian. 2020. Einsame Wölfe. Der neue Terrorismus rechter Einzeltäter, Schriftenreihe der Bundeszentrale für Politische Bildung. Bonn: Bundeszentrale für Politische Bildung.

Hißnauer, Christian, Stefan Scherer, und Claudia Stockinger. 2014. Zwischen Serie und Werk: Fernsehund Gesellschaftsgeschichte im Tatort. Einleitung. In Zwischen Serie und Werk. Fernseh- und Gesellschaftsgeschichte im „Tatort“, Hrsg. Christian Hißnauer, Stefan Scherer, und Claudia Stockinger, 7-25. Bielefeld: transcript.

Höcker, Charlotte, Gert Pickel, und Oliver Decker. 2020. Antifeminismus - das Geschlecht im Autoritarismus? Die Messung von Antifeminismus und Sexismus in Deutschland auf der Einstellungsebene. In Autoritäre Dynamiken. Alte Ressentiments - neue Radikalität. Leipziger Autoritarismus Studie 2020, Hrsg. Oliver Decker und Elmar Brähler, 249-282. Gießen: Psychosozial.

Höppner, Jana. 2020. Der rechte Hass auf Frauen. Deutsche Welle (DW), 27. Juli 2020. https://www.dw. com/de/der-rechte-hass-auf-frauen/a-54303776. Zugegriffen: 30. Sept. 2021.

Jäger, Siegfried. 2004. Kritische Diskursanalyse. Münster: Unrast.

John, Barbara (Hrsg.). 2014. Unsere Wunden kann die Zeit nicht heilen. Was der NSU-Terror für die Opfer und Angehörigen bedeutet. Breisgau: Herder.

Kaiser, Susanne. 2020. Politische Männlichkeit. Wie Incels, Fundamentalisten und Autoritäre für das Patriarchat mobil machen. Berlin: Suhrkamp.

Kaiser, Susanne. 2021. Incels-Expertin: „Die Atmosphäre in diesen Foren ist düster“. Der Standard. https:// www.derstandard.de/story/2000125380956/incels-expertin-die-atmosphaere-in-diesen-foren-istduester. Zugegriffen: 30. Sept. 2021.

Karakayali, Juliane, Çagri Kahveci, Doris Liebscher, und Carl Melchers (Hrsg.). 2017. Den NSU-Komplex analysieren. Bielefeld: transcript.

Kimmel, Michael. 2013. Angry White Men: American Masculinity at the End of an Era. New York: Nation Books.

Kleffner, Heike. 2015. Sozialarbeit und der NSU-Komplex. Aus Politik und Zeitgeschichte (APuZ) 65(40):44-48.

Koch, Matthias. 2020. Einsam, krank, gefährlich: Im Incel-Kopf von Tobias R. Redaktionsnetzwerk Deutschland (RND), 22. Februar 2020. https://www.rnd.de/politik/einsam-krank-gefahrlich-imincel-kopf-von-tobias-r-F4RLHLV3QRFRXANIHN6U7H4SVQ.html. Zugegriffen: 30. Sept. 2021.

Kopke, Christoph. 2017. Amoktat, Attentat, Hasskriminalität? Überlegungen zur Bewertung des mehrfachen Mordes des David S. https://www.muenchen.de/rathaus/dam/jcr:95fb8762-ff86-4ece-ad53b4cc9e114837/2017-10-06\%20Gutachten\%20Hintergr\%C3\%BCnde\%20und\%20Folgen\%20des \%20OEZ-Attentats\%20Kopke.pdf. Zugegriffen: 30. September 2021.

Köttig, Michaela. 2013. Rechtsextremer Terror NSU. Die Konstruktion von Geschlechterstereotypen. In NSU Terror. Ermittlungen am rechten Abgrund. Ereignis, Kontexte, Diskurse, Hrsg. Imke Schmincke, und Jasmin Siri, 155-166. Bielefeld: Transcript.

Kracher, Veronika. 2020a. Im Krieg gegen Frauen. Incels verlagern ihren Selbsthass auf andere. In Rechte Ego-Shooter. Von der virtuellen Hetze zum Livestream-Attentat, Hrsg. Jean-Philipp Baeck, und Andreas Speit, 68-86. Bonn: Sonderausgabe für die Bundeszentrale für politische Bildung.

Kracher, Veronika. 2020b. Incels. Geschichte, Sprache und Ideologie eines Online-Kults. Mainz: Ventil.

Lang, Juliane. 2015. Familie und Vaterland in der Krise. Der extrem rechte Diskurs um Gender. In Sabine Hark, und Paula-Irene Villa, Hrsg. Anti-Genderismus. Sexualität, Geschlecht als Schauplätze aktueller politischer Auseinandersetzungen, 167-181. Bielefeld: transcript.

Lückenlos e. V., Hrsg. 2020. Tribunale NSU-Komplex auflösen: Wir klagen an! Leipzig: Eigenverlag.

Madjlessi-Roudi, Sara. 2018. Unsäglicher Rassismus. Wie die Köln-Debatte den politischen Diskurs im Land verändert hat. In In Durchmarsch von rechts, Hrsg. Friedrich Burschel, 3. Aufl., 156-167. Berlin: Rosa-Luxemburg-Stiftung.

Manne, Kate. 2019. Down Girl. Die Logik der Misogynie. Bonn: Sonderausgabe für die Bundeszentrale für politische Bildung.

Meltzer, Christine E. 2021. Tragische Einzelfälle? Wie Medien über Gewalt gegen Frauen berichten. OBS-Arbeitspapier 47. https://www.otto-brenner-stiftung.de/fileadmin/user_data/stiftung/02_ 
Wissenschaftsportal/03_Publikationen/AP47_Tragische_Einzelfaelle.pdf. Zugegriffen: 30. Sept. 2021.

Meuser, Michael. 2010. Geschlecht und Männlichkeit. Soziologische Theorie und kulturelle Deutungsmuster, 3. Aufl., Wiesbaden: VS.

Nagle, Angela. 2018. Die digitale Gegenrevolution. Online-Kulturkämpfe der Neuen Rechten von 4chan und Tumblr bis zur Alt-Right und Trump. Bielefeld: transcript.

NSU-Watch. 2020. Aufklären und Einmischen. Der NSU-Komplex und der Münchener Prozess. Berlin: Verbrecher-Verlag.

Puls, Hendrik. 2020. „Gamification des Terrors“. Ein brauchbarer Begriff um rechtsterroristische Anschläge zu beschreiben? Working Paper. http://www.nfg-rexdel.de/images/Working_Paper_3.pdf. Zugegriffen: 8. Juli 2021.

Quent, Matthias. 2016. Rassismus, Radikalisierung, Rechtsterrorismus. Wie der NSU entstand und was er über die Gesellschaft verrät. Weinheim und Basel: Beltz Juventa.

Quent, Matthias. 2017. Ist die Mehrfachtötung am OEZ München ein Hassverbrechen? https://www.idzjena.de/fileadmin/user_upload/Gutachten_OEZ_M\%C3\%BCnchen_MQuent.pdf. Zugegriffen: 30. Sept. 2021.

Röpke, Andrea. 2020. Terror von rechts. Militante Kontinuität und politische Ignoranz. In Rechte EgoShooter. Von der virtuellen Hetze zum Livestream-Attentat, Hrsg. Jean-Philipp Baeck, und Andreas Speit, 108-131. Bonn: Sonderausgabe für die Bundeszentrale für politische Bildung.

Schmincke, Imke, und Jasmin Siri (Hrsg.). 2013. NSU Terror. Ermittlungen am rechten Abgrund. Ereignis, Kontexte, Diskurse. Bielefeld: transcript.

Virchow, Fabian, Tanja Thomas, und Elke Grittmann. 2015. „Das Unwort erklärt die Untat,.. Die Berichterstattung über die NSU-Morde - eine Medienkritik. Hrsg. Otto-Brenner-Stiftung. Frankfurt am Main.

Virchow, Fabian. 2010. Tapfer, stolz, opferbereit - Überlegungen zum extrem rechten Verständnis ,idealer Männlichkeit“. In „,Was ein rechter Mann ist... “. Männlichkeiten im Rechtsextremismus, Hrsg. Robert Claus, Esther Lehnert, und Yves Müller, 39-52. Berlin: Dietz.

Virchow, Fabian. 2013. „Rechter Terror(ismus) in Deutschland“. In NSU Terror. Ermittlungen am rechten Abgrund. Ereignis, Kontexte, Diskurse, Hrsg. Imke Schmincke, und Jasmin Siri, 71-78. Bielefeld: transcript.

Volpers, Simon. 2020. Neue rechte Männlichkeit: Antifeminismus, Homosexualität und Politik des Jack Donovan. Hamburg: Marta Press.

Weegmann, Nicole. 2021. Tatort: Borowski und die Angst der weißen Männer.

Wenzel, Eike. 2000. >Tatort< - Deutschland. Eine Einleitung. In Ermittlungen in Sachen >Tatort. Recherchen und Verhöre, Protokolle und Beweisfotos, Hrsg. Eike Wenzel, 7-18. Berlin: Bertz. 